\title{
Educação alimentar e nutricional para população idosa: uma revisão integrativa
}

\author{
Food and nutrition education for the elderly population: an integrative review
}

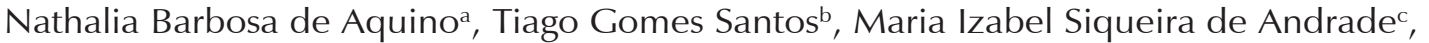 \\ Vanessa Sá Leald, Juliana Souza Oliveira ${ }^{d}$ \\ a Nutricionista. Especialista em Saúde da Família. Mestranda em Gerontologia pelo Programa de Pós-Graduação em Gerontologia da Universidade Federal de Pernambuco (UFPE) \\ bNutricionista pela UFPE/CAV. \\ c Nutricionista. Mestre em Nutrição. Doutoranda em Nutricão pelo Programa de Pós-Graduação em Nutrição da UFPE. \\ ${ }^{d}$ Nutricionista. Doutora em Nutrição. Professora Adjunta do Curso de Nutrição da UFPE/CAV.
}

\begin{tabular}{l|l} 
RESUMO Introdução: Pesquisas tem mostrado o acelerado crescimento populacional dos idosos e, a alimentação saudável é &
\end{tabular} um importante meio de alcançar um envelhecimento ativo e saudável.

Objetivo: Descrever as evidências científicas publicadas na literatura acerca das publicações na área de EAN voltada para a população idosa.

Materiais e Métodos: Revisão integrativa feita nas bases de dados eletrônicas (SCIELO, MEDLINE e LILACS), utilizando os termos: "educação alimentar e nutricional", "educação em saúde" e "idosos". Foram incluídos artigos publicados no período de 2006 a 2016, nos idiomas inglês, espanhol e português, que abordavam a temática.

Resultados: Na amostra final permaneceram 5 artigos, sendo 1 nacional e 4 internacionais. Verificou-se que os artigos analisados utilizaram como prática de EAN, atividades culinárias e lúdico-pedagógicas, três estudos também realizaram a avaliação do estado nutricional como meio de avaliação da intervenção, além de questionários e diário de campo. As pesquisas mostraram os benefícios da EAN na população idosa.

Conclusão: A educação alimentar e nutricional pode contribuir não somente para a alimentação saudável, mas inclusive, para a melhora da saúde mental e da socialização da pessoa idosa, contribuindo para o prolongamento de uma velhice com autonomia e independência.

Palavras-chave: educação alimentar e nutricional; educação em saúde; idosos.

\begin{tabular}{l|l} 
ABSTRACT Introduction: Research has shown the accelerated population growth of the elderly, and healthy eating is an important means of
\end{tabular} achieving active and healthy aging.

Objective: To describe the scientific evidence published in the literature about publications in the food and nutritional education (FNE) area for the elderly population.

Materials and Methods: An integrative review was performed in the electronic databases (SCIELO, MEDLINE and LILACS) using the terms "food and nutrition education", "health education" and "seniors". Articles published in the period 2006 to 2016 were included in the English, Spanish and Portuguese languages, which approached the theme.

Results: In the final sample, 5 articles remained, 1 national and 4 international. It was verified that the analyzed articles used as an FNE practice culinary and recreational activities, 3 studies also carried out the evaluation of nutritional status as a form of evaluating the intervention, as well as questionnaires and field diary. Research has shown the benefits of FNE in the elderly population.

Conclusion: FNE can contribute not only to healthy eating, but also to improving the mental health and socialization of the elderly, contributing to aging with autonomy and independence.

Keywords: food and nutrition education; health education; elderly. 


\section{INTRODUÇÃO}

Segundo o Relatório Mundial do Envelhecimento', em meio aos desafios desta época, que podem ser devido às mudanças climáticas e/ou às doenças infecciosas emergentes, uma tendência é garantida nesta atualidade: $\mathrm{o}$ envelhecimento das populações está acelerando rapidamente em todo o mundo. A maioria das pessoas pode esperar viver além dos 60 anos e, os efeitos disso para o sistema de saúde serão profundos.

Estudo feito por Küchemann et al. ${ }^{2}$ mostra que o Brasil acompanha essa tendência mundial, envelhecendo a passos extensos, de modo que desde a década de 40, é na população idosa que se observa as taxas mais altas de crescimento populacional e, ainda em 1950, a taxa de crescimento dessa população alcançou números superiores a $3 \%$ ao ano nessa década. Quando se compara, em um intervalo de 25 anos (1980 a 2005), o crescimento da população idosa com o crescimento da população total, constata-se que o crescimento desse público foi de $126,3 \%$, enquanto que o crescimento da população total foi de 55,3\%.

Além disso, vale ressaltar as importantes alterações fisiológicas que ocorrem durante o envelhecimento, que podem levar à redução da capacidade funcional, tornando o idoso mais vulnerável à fragilidade e à dependência de cuidados ${ }^{3}$. Essas limitações podem ser superadas, se os indivíduos adquirirem hábitos de vida saudáveis, participarem da vida em sociedade e tiverem direito à segurança, porém, é preciso considerar que essas condições dependem também das oportunidades disponibilizadas pelo contexto político e social ${ }^{4}$.

Farias et al. ${ }^{4}$ reportou que durante a Assembleia Mundial de Envelhecimento, a Organização Mundial da Saúde (OMS) apresentou o Plano Internacional de Ações sobre o Envelhecimento (PIAE), que tinha como uma de suas diretrizes, a implementação de estratégias com objetivo de alcançar o envelhecimento ativo. Nascendo então, a proposta de envelhecimento ativo, uma política de saúde composta por um grupo de sete determinantes: comportamentais, pessoais, ambiente físico, ambiente social, econômico, sistemas de saúde e serviço social, cultura e gênero.

É importante salientar também, que um dos pontos abordados na Política de Envelhecimento Ativo (2005) é a alimentação saudável ${ }^{5}$. Estudo feito por Ramos et al. ${ }^{6}$ mostra que a educação alimentar e nutricional (EAN) é importante para promoção de hábitos alimentares saudáveis, sendo recomendada pelas políticas públicas de alimentação e nutrição. O que torna necessária a prática e o desenvolvimento de ações de EAN para esse público, já que devido às mudanças decorrentes da globalização, como a inserção da mulher no mercado de trabalho e o ritmo de vida acelerado do mundo capitalista, existem poucas práticas alimentares saudáveis.

Assim, fazem-se necessários estudos que promovam ações de educação alimentar e nutricional com idosos, dessa maneira, o objetivo deste estudo foi descrever as evidências científicas publicadas na literatura acerca das publicações na área de EAN voltada para a população idosa.

\section{MATERIAIS E MÉTODOS}

Esta pesquisa trata-se de uma revisão integrativa, cuja finalidade é reunir e sintetizar resultados de pesquisas sobre um tema delimitado ou questão, colaborando para o aprofundamento do conhecimento do tema pesquisado ${ }^{7}$.

Para a estruturação desta pesquisa foram determinadas as sequentes etapas metodológicas: estabelecimento da questão norteadora; determinação de critérios para inclusão e exclusão dos artigos; avaliação dos estudos pré-selecionados; interpretação e discussão dos resultados e apresentação da revisão integrativa ${ }^{7}$.

A pesquisa aconteceu nos meses de junho e julho de 2016 e para realização da primeira etapa foi elaborada a seguinte questão norteadora para o estudo: Quais as evidências científicas publicadas nos últimos dez anos que identificaram as práticas de EAN na população idosa? A definição deste espaço de tempo fundamenta-se pela baixa disponibilidade de estudos relacionados à temática com abordagem voltada para os idosos.

A próxima etapa constituiu-se pela seleção dos artigos, por meio de busca das publicações da literatura científica, no período de janeiro de 2006 a junho de 2016 nos idiomas inglês, espanhol e português, nas bases de dados da Biblioteca Virtual em Saúde (BVS) e da Scielo (Scientific Electronic Library Online). Na base de dados BVS foi possível realizar uma busca simultânea das publicações relevantes nas principais bases de dados científicos no campo nacional e internacional: Lilacs (Literatura Latino-Americana e do Caribe em Ciências da Saúde) e Medline (Medical Literature Analysis and Retrieval System Online).

Os critérios de inclusão estabelecidos para a elegibilidade dos artigos foram: ser artigo original; responder a questão norteadora; ter disponibilidade eletrônica na forma de texto completo, ter sido publicado no período mencionado nos idiomas português, espanhol ou inglês. Foram excluídos: estudos repetidos em uma ou mais bases de dados, artigos de revisão, dissertações, teses, editoriais de jornais sem caráter científico, bem como os artigos que não tivessem a aprovação do Comitê de Ética em Pesquisa.

Para a busca dos artigos foram utilizadas três palavraschave identificadas nos Descritores em Ciências da Saúde (DeCS) e no Mesh (Medical Subject Headings): "educação 
alimentar e nutricional", "educação em saúde" e "idosos", sendo realizados os seguintes cruzamentos entre palavraschave e operadores boleanos: "educação alimentar e nutricional" AND "educação em saúde" AND "idosos", na base de dados BVS para busca simultânea na Lilacs e Medline, depois a troca do $A N D$ por $O R$, ficando o cruzamento da seguinte maneira: "educação alimentar e nutricional" $O R$ "educação em saúde" AND "idosos", na base de dados Scielo. Com isso, foram encontradas 142 publicações $($ Scielo $=15$, Lilacs $=6$, Medline $=121)$.

A seleção dos artigos foi realizada a partir da leitura dos títulos e resumos dos artigos, excluindo-se aqueles que não atendiam aos critérios de inclusão. Em seguida, foi realizada a leitura na íntegra dos artigos eleitos, a fim de coletar os dados e analisar o rigor metodológico.

Para uma melhor avaliação do rigor metodológico e dos níveis de evidência de cada artigo incluído nesta revisão integrativa, foram utilizados dois instrumentos: o primeiro, adaptado do Critical Apppraisal Skills Programme (CASP) Programa de habilidades em leitura crítica, integrante do "Public Health Resource Unit" (PHRU)8, ${ }^{8,9}$. O instrumento é composto por 10 itens (máximo 10 pontos), envolvendo: 1) objetivo; 2) adequação metodológica; 3) apresentação dos procedimentos teóricos e metodológicos; 4) seleção da amostra; 5) procedimento para a coleta de dados; 6) relação entre o pesquisador e pesquisados; 7) consideração dos aspectos éticos; 8) procedimento para a análise dos dados; 9) apresentação dos resultados; 10) importância da pesquisa. Os estudos foram classificados de acordo com as seguintes pontuações: 6 a 10 pontos (boa qualidade metodológica e viés reduzido), e mínima de 5 pontos (qualidade metodológica satisfatória, porém, com risco de viés aumentado). Neste estudo, optou-se por utilizar apenas os artigos classificados de 6 a 10 pontos.

O segundo instrumento utilizado correspondeu à Classificação Hierárquica das Evidências para Avaliação dos Estudos $^{10}$, e contempla os seguintes níveis: I) revisão sistemática ou metanálise; II) ensaios clínicos randomizados; III) ensaio clínico sem randomização; IV) estudos de coorte e de casocontrole; V) revisão sistemática de estudos descritivos e qualitativos; VI) único estudo descritivo ou qualitativo; VII) opinião de autoridades e/ou relatório de comitês de especialidades. Nesta pesquisa foram excluídos os artigos com nível de evidência I, por se tratar de revisão. Ao final da análise destes dois instrumentos, permaneceram para análise, cinco artigos.

\section{RESULTADOS}

Realizados os cruzamentos entre os descritores, foram encontrados 142 artigos, os quais passaram por uma pré-seleção mediante a leitura dos títulos e dos resumos, quando necessário. Ao término dessa fase foram pré-selecionados 15 artigos, dos quais 2 estavam duplicados, sendo 13 selecionados e lidos na íntegra de modo a identificar à adequação dos mesmos aos critérios de inclusão destinados a esta revisão, concluídas as etapas de seleção dos artigos, chegou-se à amostra final de 5 artigos, apresentados na Tabela 1 e, que apresentaram escore acima de 6 no rigor metodológico proposto por Milton ${ }^{8}$. A Figura 1 apresenta o fluxograma dos artigos encontrados, assim como os motivos da exclusão dos estudos.

Na base de dados da Medline foram encontrados 4 artigos que se enquadraram nos critérios de inclusão deste estudo, todos foram publicados na língua inglesa entre os anos de 2013 e 2015. Na Scielo, foi incluído apenas um artigo, de língua portuguesa, publicado em 2008, e na Lilacs, nenhum dos artigos avaliados estava de acordo com os critérios de inclusão.

Quanto à origem das publicações, foi selecionado um artigo brasileiro e quatro artigos internacionais, dos quais dois foram provenientes dos Estados Unidos, um da Malásia e por fim, um da China. Com relação ao desenho metodológico dos artigos selecionados, identificou-se que destes, um aplicou como método o delineamento qualitativo-descritivo, um utilizou o método quase-experimental, dois estudos o desenho experimental e um estudo randomizado controlado. Dessa forma, em relação à força das evidências ${ }^{10}$ obtidas nos artigos, foi encontrado um artigo com nível II ${ }^{15}$, um artigo com nível III ${ }^{12}$, dois com nível IV ${ }^{13,14}$ e um com nível de evidência VI ${ }^{11}$.

Observou-se um baixo número de publicações na área de EAN voltada para o público idoso, especialmente estudos de intervenção. No tocante às ações adotadas, três dos estudos além de aconselhamento sobre alimentação também realizaram atividades culinárias entre os idosos ${ }^{12,13,14}$, um deles realizou estratégias lúdico-pedagógicas ${ }^{11}$, com materiais educativos, cartazes, produtos alimentícios, pirâmide dos alimentos e outro aplicou o uso da música como meio de aprendizado entre a população estudada ${ }^{15}$.

O tempo de duração das atividades variou entre um ano e quinze dias, já a avaliação da metodologia das intervenções foi feita através da análise do conhecimento dos idosos sobre nutrição por meio de questionários ${ }^{13,14,15}$, um dos estudos fez a partir de correlações entre os dados das entrevistas, o relatório das aulas lúdico-pedagógicas e as anotações do diário de campo ${ }^{11}$, e, três dos artigos, também realizaram a avaliação do estado nutricional|12,13,14, sendo essas avaliações realizadas antes e após as intervenções.

No que diz respeito aos principais resultados encontrados nos estudos avaliados, foram observadas mudanças do conhecimento em nutrição e nas escolhas alimentares saudáveis e, entre os que realizaram avaliação do estado nutricional, houve melhora significativa entre os participantes. 
Tabela 1. Distribuição dos artigos segundo ano de publicação/autores, tipo de estudo, nível de evidência, país/idioma, temática e principais resultados.

\begin{tabular}{|c|c|c|c|c|c|c|c|c|}
\hline $\begin{array}{l}\text { Ano de Publicação/ } \\
\text { Autores }\end{array}$ & $\begin{array}{l}\text { Base de } \\
\text { Dados }\end{array}$ & Periódico & Tipo de Estudo & n & $\begin{array}{l}\text { Nível de } \\
\text { Evidência }\end{array}$ & $\begin{array}{l}\text { País/ } \\
\text { Idioma }\end{array}$ & Temática & Principais Resultados \\
\hline $\begin{array}{l}\text { 2008/ } \\
\text { Alencar et al. }{ }^{11}\end{array}$ & Scielo & Revista de Nutrição & $\begin{array}{l}\text { Qualitativo } \\
\text { descritivo }\end{array}$ & 19 & VI & $\begin{array}{l}\text { Brasil/ } \\
\text { Português }\end{array}$ & $\begin{array}{l}\text { A pesquisa analisa a contribuição } \\
\text { da educação nutricional a partir } \\
\text { das suas dimensões sócio-políticas } \\
\text { que permeiam a promoção da } \\
\text { saúde }\end{array}$ & $\begin{array}{l}\text { As oficinas lúdico-pedagógicas } \\
\text { proporcionaram aos idosos além } \\
\text { do aprendizado sobre alimentação, } \\
\text { a interação social, bem como o } \\
\text { cuidado com a saúde. }\end{array}$ \\
\hline $\begin{array}{l}\text { 2013/ } \\
\text { Shahar et al. }{ }^{12}\end{array}$ & Medline & $\begin{array}{l}\text { Public Health } \\
\text { Nursing }\end{array}$ & Quase-experimental & 47 & III & $\begin{array}{l}\text { Malásia/ } \\
\text { Inglês }\end{array}$ & $\begin{array}{l}\text { O estudo teve como objetivo } \\
\text { determinar a eficácia da } \\
\text { educação nutricional (sessões } \\
\text { de aconselhamento em grupo, } \\
\text { palestras, culinária e demonstrações } \\
\text { de exercícios) na melhoria dos } \\
\text { indicadores antropométricos, } \\
\text { clínicos e bioquímicos da rurais } \\
\text { malaios mais velhos com síndrome } \\
\text { metabólica (SM) }\end{array}$ & $\begin{array}{l}\text { Os homens que receberam } \\
\text { intervenção apresentaram } \\
\text { maior redução de peso corporal } \\
\text { comparado com o grupo } \\
\text { controle, também apresentaram } \\
\text { percentual de colesterol LDL-c } \\
\text { menor }(p<0.05) \text {. As mulheres } \\
\text { apresentaram menor circunferência } \\
\text { da cintura e peso corporal } \\
(p<0.01) \text {. }\end{array}$ \\
\hline $\begin{array}{l}\text { 2014/ } \\
\text { Yan Chung et al. }{ }^{13}\end{array}$ & Medline & $\begin{array}{l}\text { Asia Pacific } \\
\text { Journal of Clinical } \\
\text { Nutrition }\end{array}$ & Experimental & 60 & IV & $\begin{array}{l}\text { China/ } \\
\text { Inglês }\end{array}$ & $\begin{array}{l}\text { Avalia um programa de } 3 \text { semanas } \\
\text { compreendendo demonstrações } \\
\text { culinárias com amostras de } \\
\text { alimentos saudáveis para idosos } \\
\text { motivando para cozinhar mais e } \\
\text { melhorar seu estado nutricional }\end{array}$ & $\begin{array}{l}\text { Os participantes apresentaram } \\
\text { melhora no estado nutricional } \\
\text { ( } p=0.019 \text { ), além disso, o programa } \\
\text { de educação alimentar enfatizou } \\
\text { a transferência de conhecimentos } \\
\text { sobre as escolhas alimentares, e } \\
\text { a manutenção de habilidades de } \\
\text { cozinha para melhorar o apetite e } \\
\text { estimular as práticas de culinária dos } \\
\text { idosos, como forma de incentivá-los } \\
\text { a comer de forma mais saudável. }\end{array}$ \\
\hline $\begin{array}{l}\text { 2014/ } \\
\text { Francis et al. }{ }^{14}\end{array}$ & Medline & $\begin{array}{l}\text { Journal of Nutrition } \\
\text { in Gerontology and } \\
\text { Geriatrics }\end{array}$ & Experimental & 73 & IV & $\begin{array}{l}\text { Estados Unidos/ } \\
\text { Inglês }\end{array}$ & $\begin{array}{l}\text { O objetivo deste estudo foi } \\
\text { determinar a medida que o } \\
\text { programa CC (Chef Charles) revisto, } \\
\text { em comparação com o programa } \\
\text { CC tradicional, foi capaz de reduzir } \\
\text { o risco nutricional e melhorar o } \\
\text { consumo alimentar, segurança } \\
\text { alimentar e nutrição dos idosos. }\end{array}$ & $\begin{array}{l}\text { Redução no risco nutricional dos } \\
\text { participantes do programa, além } \\
\text { disso, foram percebidas mudanças } \\
\text { dietéticas positivas }(p<0.042) \text {, como } \\
\text { inclusão de escolhas saudáveis na } \\
\text { dieta, aumento da ingestão de frutas } \\
\text { e vegetais, bem como, diminuição } \\
\text { do consumo de doces e refrigerantes. }\end{array}$ \\
\hline $\begin{array}{l}\text { 2015/ } \\
\text { McClelland et al. }{ }^{15}\end{array}$ & Medline & $\begin{array}{l}\text { Journal of Nutrition } \\
\text { in Gerontology and } \\
\text { Geriatrics }\end{array}$ & $\begin{array}{l}\text { Randomizado } \\
\text { Controlado }\end{array}$ & 458 & II & $\begin{array}{l}\text { Estados Unidos/ } \\
\text { Inglês }\end{array}$ & $\begin{array}{l}\text { Investiga se ao cantar uma canção } \\
\text { de educação nutricional melhoraria } \\
\text { o conhecimento dos idosos sobre } \\
\text { nutrição }\end{array}$ & $\begin{array}{l}\text { O grupo de intervenção com música } \\
\text { teve impacto estatisticamente } \\
\text { significante }(p<0.05) \text { com relação ao } \\
\text { conhecimento em nutrição quando } \\
\text { comparado com o grupo controle. }\end{array}$ \\
\hline
\end{tabular}
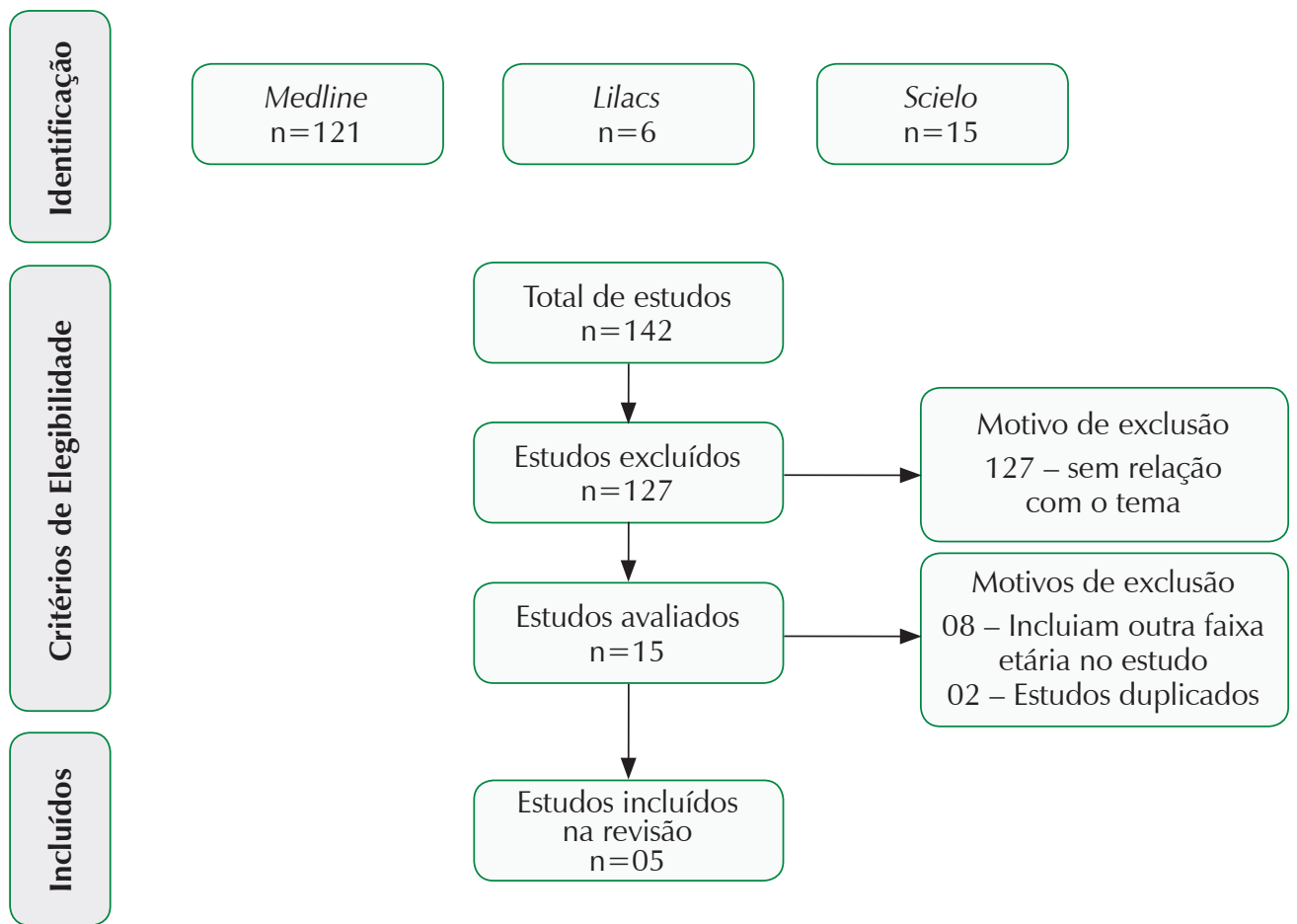

Figura 1. Fluxograma da busca e seleção dos artigos. 


\section{DISCUSSÃO}

Foi observada uma escassez de publicações com práticas de EAN, especialmente com amostras constituídas apenas da população idosa, de acordo com Santos et al. ${ }^{16}$ mesmo a EAN tendo sua relevância reconhecida, ainda há poucas referências na área atualmente, além disso, deve-se analisar que o campo da gerontologia também vem ganhando força, muito recentemente, devido à rápida transição demográfica mundial deste século, associada ao desafio dos múltiplos problemas médicos, psicossociais e econômicos gerados pela velhice $^{17}$, o que enfatiza a necessidade urgente de práticas de educação alimentar e nutricional para os idosos, tendo em vista que a alimentação saudável é um dos fatores que pode contribuir para o envelhecimento ativo ${ }^{4}$.

Três estudos realizaram como método de intervenção a prática culinária ${ }^{12,13,14} \mathrm{e}$, em dois desses, foi possível observar mudanças no estado nutricional dos participantes, segundo Castro et al. ${ }^{18}$ o cozinhar coletivo propicia o contato sensorial com os alimentos e a criação e preparação das receitas, e ainda, estimula o aprendizado com a subjetividade, envolvendo sentimentos e experiências de relação com a comida em diversos âmbitos.

Adicionalmente, Diez-Garcia et al. ${ }^{19}$ mostra em seu estudo que a prática culinária proporciona motivação, reflexão, ampliação da consciência sobre sua relação com a comida no cotidiano, aprendizado conceitual e práticas alimentares mais saudáveis, mostrando ser uma estratégia viável para educação alimentar em diferentes públicos, de forma que essa metodologia contribui até mesmo para o desenvolvimento da criticidade e autonomia do indivíduo, logo a importância de abordagens genuinamente práticas, podem tornar o aprendizado mais rápido, já que é possível materializar a teoria no ato de cozinhar, podendo ainda, promover para o indivíduo idoso uma atividade que ocupe o seu tempo, que algumas vezes, pode se apresentar ocioso.

No estudo de Alencar et al. ${ }^{11}$, as abordagens utilizadas foram atividades lúdico-pedagógicas, conforme estudo realizado por Oliveira et al. ${ }^{20}$, as atividades lúdicas propiciam diversas vantagens, além de ser uma técnica respaldada por educadores como Piaget, Montessori e George Snyders ${ }^{21}$. Segundo estudo feito por Macedo et al. ${ }^{22}$ e Oliveira et al. ${ }^{20}$, a ludicidade pode ser considerada uma necessidade do ser humano em qualquer idade e não pode ser vista apenas como diversão. Dentre os aspectos positivos da atividade lúdica estão: simplificação da aprendizagem, o desenvolvimento pessoal, social e cultural, contribui para uma boa saúde mental, capacita para um estado interior fértil, favorece os processos de socialização, comunicação, expressão e construção do conhecimento ${ }^{20,21,22}$, ou seja, a técnica lúdico-pedagógica pode contribuir não somente para alimentação saudável, mas, sobretudo, como um fator de integração social e de melhoramento cognitivo do indivíduo idoso, corroborando de forma legítima para o envelhecimento ativo.

E, de maneira criativa, o estudo de McClelland et al. ${ }^{15}$, abordou a alimentação saudável com o público idoso mediante o uso da música, realizando paródias com músicas que tivessem sido marcantes no tempo de juventude do público alvo, pesquisa feita por Marques et al. ${ }^{23}$, mostrou que a musicoterapia com o indivíduo idoso incentiva o prazer de criar e partilhar a música, aplicando atividades como cantar, dançar e recordar canções que fazem parte de sua história de vida, retomando características positivas da mesma e garantindo a sua confiança e identidade, assim, a metodologia desenvolvida pela música ${ }^{15}$, além de ensinar sobre alimentação também pode colaborar para o melhoramento da memória, bem como a qualidade de vida dos idosos.

No que diz respeito aos critérios de avaliação adotados, três das pesquisas analisadas ${ }^{13,14,15}$, adotaram o meio de questionário antes e após a intervenção, duas adicionaram a esse critério, a avaliação do estado nutricional (AEN) ${ }^{13,14}$, uma pesquisa só utilizou a $\mathrm{AEN}^{12}$ e uma fez interligações entre os dados das entrevistas, o relatório das aulas lúdicopedagógicas e as anotações do diário de campo ${ }^{11}$, estudo feito por Ramos et al. ${ }^{6}$ revela a necessidade de reflexão sobre o que seja eficácia nas atividades de EAN, argumenta-se uma indispensável avaliação crítica do processo educativo, e não apenas dos resultados objetivos, tendo em vista as complexidades do processo.

Dentre os principais resultados obtidos entre os artigos selecionados, foi observado que as metodologias aplicadas não tiveram enfoque apenas em palestras, mas na abordagem prática da utilização dos alimentos, bem como atividades lúdico-pedagógicas, como a música e uso de cartazes, pincéis, cartolinas, embalagens de produtos alimentícios, pirâmide dos alimentos, dentre outros. Mostrando assim, a importância de uma educação que propicie a interação social e a reflexão crítica dos educandos, educação essa proposta por Freire ${ }^{24}$, que somado ao fato de ser de acordo com a cultura e realidade dos educandos, pode apresentar melhores resultados quando comparada com a educação bancária, sendo essa, observada no campo da alimentação através de atividades de EAN baseadas somente em palestras, em que consiste na mera transmissão de conceitos de nutrição para a população ${ }^{25}$, onde, em geral, o indivíduo não consegue associar o aprendizado com sua realidade de vida, gerando poucos resultados positivos. 
Por fim, também se faz necessário pontuar a importância da educação na vida dos idosos, estudo conduzido por Cunha et al. ${ }^{26}$ mostra que a educação tem um papel essencial na inclusão social dos idosos e aponta o seu papel fundamental na construção e desenvolvimento integral do ser humano, onde a atenção não gira em torno de preparar o ser humano para a vivência de uma velhice feliz, mas cooperar para o desenvolvimento do ser humano para uma vida harmoniosa, da qual fazem parte as várias fases do ciclo de vida, com a inclusão da última, demonstrando assim, as profundas e positivas mudanças que a prática educacional pode realizar no indivíduo, independentemente de sua faixa etária, e também, destacando sua necessidade, tendo em vista os seus benefícios.

Considerando as diferentes abordagens realizadas pelas pesquisas analisadas, nota-se que a EAN pode contribuir para muito além da alimentação saudável, colaborando inclusive, para o melhoramento da saúde mental e da socialização da pessoa idosa, gerando um prolongamento de uma velhice com autonomia e independência, sendo assim, urgente a necessidade de mais estudos de EAN, haja vista o baixo número de publicações nessa área. Ademais, são necessários estudos com diferentes metodologias e com enfoque na população idosa, especialmente devido ao acelerado crescimento mundial desse público.

\section{REFERÊNCIAS}

1. World Health Organization. Relatório Mundial de Envelhecimento e Saúde. Genebra: WHO; 2015.

2. Kuchemann BA. Envelhecimento populacional, cuidado e cidadania: velhos dilemas e novos desafios. Soc Estado. 2012; 27(1):165-80. https://doi.org/10.1590/S0102-699220120001 00010

3. Mesquita GV. Morbimortalidade em idosos por fratura proximal do fêmur. Texto Contexto Enferm. 2009;18(1):63-7. https://doi. org/10.1590/S0104-07072009000100008

4. Farias RG, Santos SMA. Influência dos determinantes do envelhecimento ativo entre idosos mais idosos. Texto Contexto Enferm. 2012;21(1):167-76. https://doi.org/10.1590/S010407072012000100019

5. World Health Organization. Envelhecimento ativo: uma política de saúde. Brasília: Organização Pan-Americana da Saúde; 2005.

6. Ramos FP, Santos LAS, Reis ABC. Educação alimentar e nutricional em escolares: uma revisão de literatura. Cad Saúde Pública. 2013;29(11):2147-61. https://doi.org/10.1590/0102$311 \times 00170112$

7. Mendes KDS, Silveira RCCP, Galvão CM. Revisão integrativa: método de pesquisa para a incorporação de evidências na saúde e na enfermagem. Texto Contexto Enferm. 2008; 17(4):758-64. https://doi.org/10.1590/S0104-070720080004 00018
8. Milton Keynes Primary Care Trust. Critical Appraisal Skills Programme. London: Oxford; 2002.

9. Critical Appraisal Skills Programme (CASP) [Internet]. 2013 [citado 2016]. Disponível em: http://www.casp-uk.net/\#!casptoolschecklists/c18f8

10. Stillwell SB, Fineout-Overholt E, Melnyk BM, Williamson KM. Evidence-based practice: step by step. Am J Nurs. 2010;110(5): 41-7. https://doi.org/10.1097/01.NAJ.0000372071.24134.7e

11. Alencar MSS, Barros Júnior FO, Carvalho CMRG. Os aportes sócio-políticos da educação nutricional na perspectiva de um envelhecimento saudável. Rev Nutr.2008;21(4):369-81. https://doi. org/10.1590/S1415-52732008000400001

12. Shahar S, Adznam SN, Lee LK, Yusof NA, Salleh M, Mohamed Sakian NI. A Nutrition Education Intervention for Anthropometric and Biochemical Profiles of Rural Older Malays with Metabolic Syndrome. Public Health Nurs. 2013;30(2):140-9. https://doi. org/10.1111/j.1525-1446.2012.01051.x

13. Yan Chung LM, Yee Chung JW. Effectiveness of a food education program in improving appetite and nutritional status of elderly adults living at home. Asia Pac J Clin Nutr 2014;23(2):315-20

14. Francis SL, Macnab L, Shelley M. A theory-based newsletter nutrition education program reduces nutritional risk and improves dietary intake for congregate meal participants. J Nutr Gerontol Geriatr. 2014;33(2):91-107 https://doi.org/10.1080/21551197.2014. 906336

15. McClelland JW, Jayaratne KS, Bird C. Use of song as an effective teaching strategy for nutrition education in older adults. J Nutr Gerontol Geriatr. 2015;34(1):22-33 https://doi.org/10.1080/2155 1197.2014.998327

16. Santos LAS. Educação alimentar e nutricional no contexto da promoção de práticas alimentares saudáveis. Rev Nutr. 2005,18(5):681-92. https://doi.org/10.1590/S1415-52732005 000500011

17. Rosset I, Pedrazzi EC, Roriz-Cruz M, Morais EP, Rodrigues RAP. Tendências dos estudos com idosos mais velhos na comunidade: uma revisão sistemática (inter)nacional. Rev Esc Enferm USP. 2011;45(1):264-71. https://doi.org/10.1590/S008062342011000100037

18. Castro IRR, Souza TSN, Maldonado LA, Caniné ES, Rotenberg S, Gugelmin SA. A culinária na promoção da alimentação saudável: delineamento e experimentação de método educativo dirigido a adolescentes e a profissionais das redes de saúde e de educação. Rev Nutr. 2007;20(6):571-88. https://doi.org/10.1590/S1415-5273 2007000600001

19. Diez-Garcia RW, Castro IRR. A culinária como objeto de estudo e de intervenção no campo da alimentação e nutrição. Cien Saude Colet. 2011;16(1):91-8. https://doi.org/10.1590/S141381232011000100013

20. Oliveira AJ, Raffaelli SC, Colauto RD, Casa Nova SP. Estilos de aprendizagem e estratégias ludopedagógicas: percepções no ensino da contabilidade. Adv Sci Appl Account. 2013;6(2):236-62. https:// doi.org/10.14392/ASAA.2013060206

21. Almeida PN. Educação lúdica: técnicas e jogos pedagógicos. São Paulo: Loyola; 1990. 
22. Macedo L, Petty ALS, Passos NC. Os jogos e o lúdico na aprendizagem escolar. Porto Alegre: Artmed; 2005.

23. Marques MHPG. Comunicar com o coração: a musicoterapia e o idoso com doença de Alzheimer. Relatório de estágio do Mestrado em Musicoterapia. Lisboa: Universidade Lusíada; 2013.

24. Freire P. Pedagogia do Oprimido. 17aㅡ ed. Rio de Janeiro: Paz e Terra; 1987.
25. Rodrigues LPF, Roncada MJ. Educação nutricional no Brasil: evolução e descrição de proposta metodológica para escolas. Comun Ciênc Saúde. 2008;19(4):315-22.

26. Cunha L, Lopes MSL, Pereira F. Papel da educação para a promoção do suporte social e nível de satisfação com a vida. In: López ST, Pérez OG, Calvo JVP. La crisis social y el del bienestar las respuestas de la pedagogía social. Oviedo: Universidad de Oviedo; 2013. p. 228-35. 